\title{
Microfibrillar-associated protein 5 is linked with markers of obesity-related extracellular matrix remodeling and inflammation
}

\author{
M Vaittinen ${ }^{1}$, M Kolehmainen ${ }^{1}, \mathrm{U} \mathrm{Schwab}^{1,2}, \mathrm{M}$ Uusitupa $^{1,3}$ and L Pulkkinen ${ }^{1}$ \\ ${ }^{1}$ Institute of Public Health and Clinical Nutrition, Department of Clinical Nutrition, Food and Health Research Centre, \\ University of Eastern Finland, Kuopio, Finland; ${ }^{2}$ Institute of Clinical Medicine, Internal Medicine, Kuopio University \\ Hospital, Kuopio, Finland and ${ }^{3}$ Research Unit, Kuopio University Hospital, Kuopio, Finland
}

\begin{abstract}
Objective: Microfibrillar-associated protein 5 (MFAP5) is an extracellular matrix (ECM) glycoprotein, which is colocalized with microfibrils in elastin networks. Its function in adipose tissue (AT) is not known. We have recently shown that the expression of MFAP5 is downregulated in AT along with weight reduction (WR) in persons with metabolic syndrome (MetS). The aim of this work was to study whether the change of MFAP5 mRNA expression in response to WR is associated with markers of adiposity, glucose metabolism and insulin resistance in human AT.
\end{abstract}

Design: Weight reduction intervention study in parallel study design (The Genobin study). Altogether 46 obese subjects with impaired glucose tolerance and features of MetS were randomized to a WR $(n=28)$ or a control group $(n=18)$ lasting for 33 weeks.

Measurements: Circulating glucose and insulin concentrations were measured and subcutaneous AT biopsies were performed before and after the intervention. The mRNA expression was studied by quantitative real-time PCR (QPCR).

Results: QPCR of human AT biopsy samples confirmed that MFAP5 is highly expressed in AT and its expression is decreased during WR. The mRNA expression of MFAP5 correlated positively with body mass index, and the change in MFAP5 mRNA expression during WR correlated positively with the change of body fat mass. Furthermore, the MFAP5 mRNA expression correlated negatively with circulating fasting concentrations of adiponectin and interleukin (IL)-1 $\beta$ and positively with leptin, insulin and IL-1Ra levels. In addition, the MFAP5 mRNA expression correlated positively with the mRNA expressions of peroxisome proliferator-activated receptor gamma, cyclin D2 and A disintegrin and metalloproteinase domain 12, genes involved in AT remodeling.

Conclusion: This study demonstrates that MFAP5 is highly expressed in human AT and is correlated with markers of insulin resistance. Furthermore, it is possible that MFAP5 is related to ECM remodeling during development of obesity.

Nutrition and Diabetes (2011) 1, e15; doi:10.1038/nutd.2011.10; published online 29 August 2011

Keywords: MFAP5; adipose tissue; insulin resistance; ECM remodeling

\section{Introduction}

Obesity is a chronic low-grade inflammatory state, which is characterized by an increase in circulating inflammatory factors partly due to changes in cytokine and adipokine production in adipose tissue (AT). ${ }^{1}$ In addition to mature adipocytes, AT is composed of different cell types in the stromavascular fraction ${ }^{1}$ that may have different roles in obesity-related inflammation of AT. It has been suggested

Correspondence: $\operatorname{Dr} M$ Vaittinen, Institute of Public Health and Clinical Nutrition, Department of Clinical Nutrition, Food and Health Research Centre, University of Eastern Finland, PO Box 1627, 70211 Kuopio, Finland. E-mail: maija.vaittinen@uef.fi

Received 4 April 2011; revised 10 June 2011; accepted 24 June 2011 that a proinflammatory state with a concomitant upregulation of inflammation-related genes might lead to changes in the expression of genes linked to the extracellular matrix (ECM) in order to accommodate the growing $\mathrm{AT}^{2}$ Weight reduction (WR) is an effective way to reverse the state of inflammation and decrease biological markers of inflammation in the circulation, and also the expression of inflammatory markers in AT. $^{3}$

Genome-wide transcriptomics analysis performed from AT of the subjects with metabolic syndrome (MetS) participating in the Genobin study ${ }^{4}$ showed that the ECM-associated gene, microfibrillar-associated protein 5 (MFAP5), ${ }^{5}$ also known as MAGP2, was one of the genes, whose expression was downregulated in subcutaneous AT along with WR and improved insulin sensitivity. It was found that MFAP5 mRNA 
was highly expressed in AT, although its function in human AT is not known. In this work, our aim was to investigate whether the change of MFAP5 gene expression, along with WR, is correlated with the measures of glucose metabolism and body adiposity in the Genobin study individuals and with circulating adipokines and expression of genes, which were changed after WR.

\section{Materials and methods}

Altogether 75 overweight/obese (body mass index (BMI) $28-40 \mathrm{~kg} \mathrm{~m}^{-2}$ ) subjects aged $40-70$ years were recruited into the Genobin study (NTC00621205) described in detail earlier. ${ }^{4}$ The subjects had impaired fasting glucose or impaired glucose tolerance, and at least two other features of the MetS. ${ }^{6}$ Subjects were randomly assigned to one of the following groups: WR $(n=28)$, aerobic exercise training $(n=15)$, resistance exercise training $(n=14)$ or control group $(n=18),{ }^{4}$ but in this study only the data from the WR and control groups were included and analyzed. The number of study participants varied among the correlation analyses due to missing values of the variables in some of the participants. In addition, mRNA samples were not available from all the participants. The final number of participants included in the data analysis is indicated in the table footnote. Subjects were matched for age, gender, BMI and the status of glucose metabolism. Briefly, the WR group had an intensive WR period lasting for 12 weeks during which the study subjects followed detailed instructions given by a clinical nutritionist. These instructions were based on a 4-day dietary record and an interview. Between weeks 12 and 33, the aim was to maintain the achieved reduction in weight. The control group was asked not to change their lifestyle habits. The intervention was performed in accordance with the standards of the Declaration of Helsinki. The Ethics Committee of the district hospital region of Northern Savo and Kuopio University Hospital approved the study plan. All participants gave a written informed consent.

Glucose metabolism was measured using an oral glucose tolerance test and by frequently sampled intravenous glucose tolerance test, according to the Minimal Model method, ${ }^{7}$ at baseline and at the end of the intervention. ${ }^{4}$ Insulin sensitivity index, glucose effectiveness and acute phase insulin response to glucose were calculated by the MINMOD Millenium software. ${ }^{8}$

After an overnight fast, AT samples were taken by needle biopsy from subcutaneous abdominal AT, before and after the intervention under local anesthesia $\left(10 \mathrm{mg} \mathrm{ml}^{-1}\right.$ lidocaine) to collect $0.5-5 \mathrm{~g}$ of AT for the mRNA expression studies. AT samples were washed twice with phosphatebuffered saline and treated with RNAlater according to the instructions provided by the manufacturer (Ambion, Austin, TX, USA), and stored in $-80^{\circ} \mathrm{C}$, until used for RNA extraction. RNA extraction, cDNA synthesis and quantitative real-time PCR (QPCR) were performed as previously described in detail. ${ }^{4}$ Briefly, TRIzol method followed by purification with RNeasy Mini Kit (Invitrogen, Carlsbad, CA, USA and Qiagen, Valencia, CA, USA) were used for extracting total RNA according to the manufacturer's instructions. Nanodrop (NanoDrop Technologies, Wilmington, DE, USA) was used for measuring the RNA concentration and the A260/A280 ratio. RNA was reverse-transcribed into cDNA using High-capacity cDNA Reverse Transcription Kit (Applied Biosystems, Foster City, CA, USA) according to the instructions provided by the manufacturer. QPCR with TaqMan chemistry (Applied Biosystems) by using ABI Prism 7500 analyzer (Applied Biosystems) was used for the confirmation of microarray gene expression results. The analysis for the relative quantity of a specific gene, before and after the intervention in AT, was performed as described previously. ${ }^{4}$ Expression of target genes were normalized to cyclophilin A1 (PPIA) expression.

The clinical data were analyzed using the SPSS software for Windows version 14.0 (SPSS Inc., Chicago, IL, USA). Data are given as mean \pm s.d., unless otherwise indicated. The normality of distributions of the variables was tested with the Kolmogorov-Smirnov test, with Lilliefors significance correction. Logarithmic transformation was used to achieve normal distribution, whenever needed (indicated in tables and/or figures). General linear model for univariate analysis was used to test the difference in fold-change values of mRNA expression between the groups. Paired samples $t$-test was used for comparing the baseline and endpoint measurements within the study group. Correlation analyses were carried out using Pearson's method. Partial correlation analysis with adjustment for weight at baseline and gender was used when appropriate. The WR and control groups were combined in the correlation analysis at baseline and when studying the correlations of change values. At baseline, this was carried out because the participants who were randomized either to an intervention or a control group represents the same risk population, all having MetS. Thus, the participants were homogenous regarding the selection criteria. After the intervention, the two treatment groups were analyzed separately because of potential treatment effect. Moreover, the correlations between the change of MFAP5 mRNA expression and the changes of clinical and biochemical measures were studied when the WR and control groups were combined, but also separately, for both the groups. For the clinical and biochemical measurements, $P<0.05$ was considered as statistically significant.

\section{Results}

Values for body weight, fasting plasma glucose and serum insulin concentration were at baseline $92.1 \pm 14.9 \mathrm{~kg}$, $6.2 \pm 0.4 \mathrm{mmoll}^{-1}$ and $11.5 \pm 5.4 \mathrm{pmoll}^{-1}$, and at the end of the intervention $88.7 \pm 14.0 \mathrm{~kg}, 5.9 \pm 0.3 \mathrm{mmoll}^{-1}$ and $10.7 \pm$ 


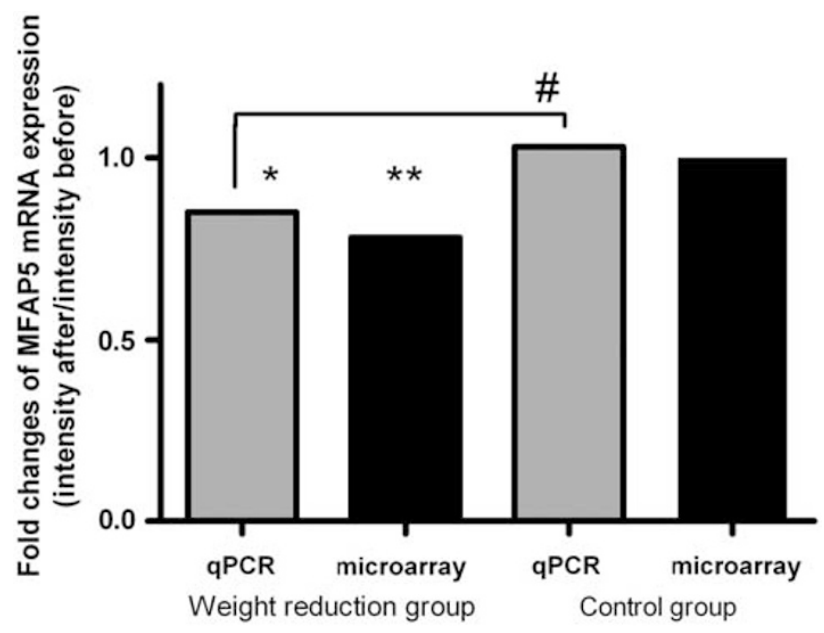

Figure 1 Fold changes of MFAP5 mRNA expression ( $y$ axis) using QPCR (gray bars) and microarray analysis (black bars) in the weight reduction group and control group. $P<0.05$ was considered statistically significant. MFAP5 gene expressed after the intervention divided by the intensity at baseline: ${ }^{\star} P=0.017,{ }^{*} P=0.004$ (comparisons were made within groups), ${ }^{\#} P=0.028$ (comparison was made between groups).

$7.0 \mathrm{pmoll}^{-1}$ for the WR group, respectively. The respective values for the control group were $87.3 \pm 8.3 \mathrm{~kg}, 6.4 \pm$ $0.6 \mathrm{mmoll}^{-1}$ and $9.9 \pm 5.0 \mathrm{pmoll}^{-1}$ at baseline and $87.7 \pm$ $9.0 \mathrm{~kg}, 6.1 \pm 0.6 \mathrm{mmoll}^{-1}$ and $10.6 \pm 6.0 \mathrm{pmoll}^{-1}$ at the end of the intervention, respectively.

The QPCR analysis for MFAP5 expression confirmed the data obtained by microarray (Figure 1). When the comparisons were made within the groups, the mRNA expression levels of MFAP5 showed significant decrease (94.2 \pm 49.0 to 81.7 $\pm 41.7 \mathrm{AU}, P=0.017)$ in the WR group and no change was seen in the control group (106.4 \pm 40.4 to $109.4 \pm$ 34.5 AU, $P=0.636)$. Similar results were also obtained by microarray showing that MFAP5 expression was significantly downregulated in the WR group $(P=0.004) .{ }^{4}$ Fold-change results by QPCR showed a significant reduction in MFAP5 mRNA expression in the WR group $(P=0.028)$ when compared with the control group.

Correlation analyses were performed with the combined study groups at baseline. MFAP5 mRNA expression correlated significantly at baseline with BMI (Table 1). Moreover, the expression of MFAP5 correlated significantly with fasting serum insulin concentration at baseline, when adjusted for baseline body weight (Table 1). There was no correlation between MFAP5 mRNA expression and fasting plasma glucose levels.

Interestingly, at baseline, the mRNA expression of MFAP5 correlated negatively with fasting serum adiponectin and positively with leptin concentrations. Moreover, a positive correlation was also found for fasting plasma interleukin (IL)-1Ra and a negative one for fasting plasma IL-1 $\beta$ concentrations, when adjusted for baseline body weight (Table 1). MFAP5 mRNA expression correlated positively with leptin gene expression (Table 1) at baseline (adjusted for baseline body weight) and with adiponectin and IL-6 expression after the WR (Table 1). However, at the expression level, there was no correlation with cytokines like transforming growth factor beta 1, tumour necrosis factor alpha, IL-1 $\beta$ or IL-1Ra (Table 1 ).

Taken into account that MFAP5 is involved in the NOTCH pathway, ${ }^{9}$ we studied the correlation of MFAP5 mRNA expression with NOTCH1 and NOTCH2 mRNA expressions. MFAP5 mRNA expression did not correlate with the expressions of these genes.

We also studied whether MFAP5 mRNA expression is correlated with expressions of genes, which are involved in modulation of AT formation. MFAP5 expression correlated positively with peroxisome proliferator-activated receptor gamma (PPAR $\gamma)$, cyclin D2 (CCND2) and a disintegrin and metalloproteinase domain 12 (ADAM12) mRNA expressions at baseline (adjusted for baseline body weight), as well as after the WR (Table 1), when adjusted for the corresponding body weight.

The change in MFAP5 mRNA expression level correlated significantly with the change IN body fat mass $(r=0.392$, $P=0.009$ ). Furthermore, the change of MFAP5 expression level correlated significantly with the changes of ADAM12, adiponectin (ADIPOQ) and NOTCH2 expression levels ( $r=0.343, P=0.026 ; r=0.433, P=0.004 ; r=0.438, P=0.006$ ), respectively, when adjusted for the change of body weight and the WR and control groups were combined. When the groups were analyzed separately, there was also a significant correlation in the WR group between the change of MFAP5 mRNA expression and the change of ADIPOQ mRNA expression. In the control group, there were no significant correlations.

\section{Discussion}

MFAP5 is associated with microfibrils in elastin networks in a number of tissues, and its function may be related to cell signaling during microfibril assembly, elastogenesis and cell survival. 5,10,11 It has been shown that MFAP5 promotes angiogenesis and interacts with NOTCH1 by either activating or suppressing its activity, depending on the cell type involved., ${ }^{9,12}$ Furthermore, MFAP5 is increased in fibrotic skin of humans and in mouse models with systemic sclerosis. ${ }^{13}$ Whether its function in AT is similar to previous findings is not known.

ECM is composed of structural and multifunctional molecules such as collagen, adhesive glycoproteins and proteoglycans. ${ }^{14,15}$ In AT, ECM maintains the structural integrity of adipocytes, and has an important role in AT formation. ${ }^{15}$ Development of obesity induces changes in AT (e.g., adipocyte hypertrophy, new adipocyte formation), which are associated with remodeling of ECM proteins and angiogenesis. ${ }^{14,15,16}$ Genes related to ECM and cytoskeleton have been shown to be upregulated by high-fat diet and 
Table 1 Correlations of MFAP5 mRNA expression in subcutaneous adipose tissue with anthropometric, biochemical measures, and with selected genes expressed in subcutaneous adipose tissue before and after the intervention in weight reduction and control groups; adjusted for body weight

\begin{tabular}{|c|c|c|c|c|c|c|}
\hline & \multicolumn{2}{|c|}{ Groups combined $(n=46)$} & \multicolumn{2}{|c|}{ Weight reduction group $(\mathrm{n}=28)$} & \multicolumn{2}{|c|}{ Control group $(\mathrm{n}=18)$} \\
\hline & \multicolumn{2}{|c|}{0 week } & \multicolumn{2}{|c|}{34 weeks } & \multicolumn{2}{|c|}{34 weeks } \\
\hline & $r^{a}$ & P-value & $r^{a}$ & P-value & $r^{a}$ & P-value \\
\hline \multicolumn{7}{|c|}{ Anthropometric and biochemical measures } \\
\hline Body mass index $\left(\mathrm{kg} \mathrm{m}^{-2}\right)^{\mathrm{b}}$ & $0.369^{\# \# \# \#}$ & 0.014 & $0.068^{\circ 0}$ & 0.737 & 0.128 & 0.613 \\
\hline Body weight $(\mathrm{kg})^{\mathrm{b}}$ & $0.043^{\# \# \#}$ & 0.785 & $-0.213^{\circ}$ & 0.297 & $-0.122^{\times}$ & 0.641 \\
\hline Body fat mass $(\mathrm{kg})^{\mathrm{b}}$ & $0.277^{\# \# \#}$ & 0.069 & $0.052^{\circ}$ & 0.798 & 0.114 & 0.653 \\
\hline Waist circumference $(\mathrm{cm})$ & $0.282^{\# \# \#}$ & 0.071 & $0.431^{\circ}$ & 0.032 & $-0.011^{\times}$ & 0.968 \\
\hline $\mathrm{S}_{\mathrm{I}}\left(\left(\mathrm{mUI}^{-1}\right)^{-1} \times \mathrm{min}^{-1}\right)$ & $-0.260^{\# \#}$ & 0.101 & $-0.162^{\circ}$ & 0.439 & $-0.429^{\times}$ & 0.017 \\
\hline $\mathrm{fS}$-adiponectin $\left(\mu \mathrm{g} \mathrm{ml}^{-1}\right)$ & $-0.378^{\# \# \#}$ & 0.014 & $-0.369^{\circ \circ}$ & 0.063 & $-0.626^{\times}$ & 0.009 \\
\hline fS-leptin $\left(\mathrm{ng} \mathrm{ml}^{-1}\right)$ & $0.361^{\# \# \#}$ & 0.019 & $0.155^{\circ}$ & 0.459 & $0.153^{\times}$ & 0.571 \\
\hline fS-insulin $\left(\right.$ pmoll $\left.{ }^{-1}\right)$ & $0.397^{\# \#}$ & 0.010 & $0.165^{\circ}$ & 0.429 & $0.352^{\times}$ & 0.181 \\
\hline fP-glucose $\left(\mathrm{mmoll}^{-1}\right)$ & $0.132^{\# \#}$ & 0.41 & $-0.093^{\circ}$ & 0.66 & $0.358^{\times}$ & 0.174 \\
\hline $\mathrm{fS}-\mathrm{TNF} \alpha\left(\mathrm{pg} \mathrm{ml^{-1 }}\right)$ & $-0.162^{\# \# \#}$ & 0.305 & $-0.296^{\circ}$ & 0.151 & $0.028^{\times}$ & 0.919 \\
\hline fP-IL1 $\beta\left(p g \mathrm{ml}^{-1}\right)$ & $-0.401^{\# \# \#}$ & 0.009 & $-0.22^{\circ}$ & 0.29 & $-0.207^{\times}$ & 0.441 \\
\hline fP-IL1 Ra $\left(\mathrm{pg} \mathrm{ml}^{-1}\right)$ & $0.347^{\# \# \#}$ & 0.024 & $0.325^{\circ}$ & 0.113 & $0.469^{\times}$ & 0.067 \\
\hline hsCRP $\left(\mathrm{mgl}^{-1}\right)$ & $-0.021^{\# \# \#}$ & 0.895 & $-0.106^{\circ}$ & 0.615 & $-0.076^{\times}$ & 0.781 \\
\hline \multicolumn{7}{|c|}{ Gene expression in adipose tissue ( $A U)$} \\
\hline Leptin & $0.338^{\# \# \#}$ & 0.028 & $0.259^{\circ}$ & 0.211 & $0.357^{\times}$ & 0.174 \\
\hline Adiponectin & $0.033^{\# \# \#}$ & 0.838 & $0.488^{\circ}$ & 0.013 & $0.011^{\times}$ & 0.968 \\
\hline PPAR- $\gamma$ & $0.511^{\# \# \#}$ & 0.001 & $0.621^{\circ}$ & 0.001 & $0.195^{\times}$ & 0.469 \\
\hline TNF- $\alpha$ & $0.132^{\# \# \#}$ & 0.404 & $0.018^{\circ}$ & 0.932 & $0.39^{\times}$ & 0.136 \\
\hline IL1- $\beta$ & $0.071^{\# \#}$ & 0.659 & $0.140^{*}$ & 0.524 & $0.09^{\times}$ & 0.741 \\
\hline IL-1Ra & $0.106^{\# \# \#}$ & 0.504 & $0.054^{*}$ & 0.807 & $0.386^{\times}$ & 0.14 \\
\hline IL-6 & $0.007^{\# \# \#}$ & 0.966 & $0.409^{\circ}$ & 0.042 & $-0.267^{\times}$ & 0.317 \\
\hline TGF- $\beta 1$ & $0.25^{\#}$ & 0.12 & $0.388^{*}$ & 0.067 & $0.089^{\times}$ & 0.743 \\
\hline NOTCH1 & $-0.277^{\#}$ & 0.083 & $-0.191^{*}$ & 0.383 & $-0.121^{\times}$ & 0.656 \\
\hline NOTCH2 & $0.191^{\#}$ & 0.239 & $0.324^{\star}$ & 0.131 & $0.37^{\times}$ & 0.158 \\
\hline CCND2 & $0.523^{\# \# \#}$ & $<0.001$ & $0.401^{\circ}$ & 0.047 & $0.392^{\times}$ & 0.133 \\
\hline ADAM22 & $0.248^{\# \# \#}$ & 0.114 & $0.261^{*}$ & 0.229 & $0.473^{\times}$ & 0.064 \\
\hline ADAM12 & $0.584^{\# \# \#}$ & $<0.001$ & $0.552^{\circ}$ & 0.004 & $0.579^{\times}$ & 0.019 \\
\hline
\end{tabular}

Abbreviations: ADAM12, A disintegrin and metalloproteinase domain 12; ADAM22, A disintegrin and metalloproteinase domain 22; AU, arbitrary unit; CCND2, cyclin D2; fP, fasting plasma; fS, fasting serum; hsCRP, high-sensitivity C reactive protein; IL-6, interleukin 6; IL-1Ra, interleukin 1 receptor antagonist; IL-1 $\beta$, interleukin 1 beta; NOTCH1, notch1 preproprotein; NOTCH2, notch2 preproprotein; PPAR $\gamma$, peroxisome proliferator-activated receptor gamma; $S_{1}$, insulin sensitivity index; TGF- $\beta 1$, transforming growth factor beta 1 ; TNF- $\alpha$, tumour necrosis factor alpha. ${ }^{a}$ Adjusted for body weight (kg). ${ }^{\mathrm{b}}$ Correlation analyses were carried

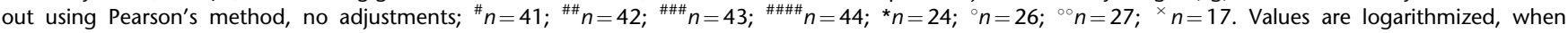
appropriate. Bold font: result statistically significant $(P<0.05)$.

correlated with body weight in mice. ${ }^{14,17}$ Not only weight gain induces ECM remodeling but also weight loss modifies the expression of ECM molecules. ${ }^{15}$ The chronic proinflammatory state in obesity is suggested to be associated with ECM remodeling and subsequent interstitial fibrosis. It has also been shown that human preadipocytes secrete fibrotic factors because of inflammatory stimulus. ${ }^{18}$

Recent human and rodent studies have demonstrated that collagen VI is highly expressed in obese state and is associated with increased BMI, adipose fat mass and inflammation. There is also evidence that a loss of collagen VI in AT leads to improvements of metabolic profile. ${ }^{19-21}$ Expression changes in microfibrillar proteins such as MFAP5, is known to participate in collagen synthesis and accumulation. ${ }^{22}$ Lemaire et al. ${ }^{23}$ have demonstrated that MFAP5 could enhance type I collagen matrix by stabilizing type I procollagen. Furthermore, it has been shown that MAGP-1, which is structurally related molecule to MFAP5, could bind to type VI collagen. It could also be possible that MFAP5 might bind to collagen VI or other ECM molecules of AT, but only fibrillin 1 has been shown to interact with MFAP5. ${ }^{23}$

Our results showed that MFAP5 expression was correlated with the change of body fat mass, fasting serum adiponectin and leptin. Reduced adiponectin levels and increased leptin levels are associated with obesity and insulin resistance. ${ }^{24}$ Moreover, adiponectin and leptin are involved in obesityrelated angiogenesis and ECM remodeling. ${ }^{16,24}$ Therefore, the found correlation between the MFAP5 gene expression and adiponectin and leptin might be related to angiogenesis and ECM remodeling during development of obesity and reduction of body fat mass along with WR.

In addition to MFAP5, also specific ADAM isoforms are shown to be involved in NOTCH pathway. Moreover, ADAM12, CCND2, NOTCH receptors and PPAR $\gamma$ are known to be involved in the development of $\mathrm{AT}$, differentiation and 
adipogenesis. ${ }^{25-27}$ Interestingly, our results showed a correlation between MFAP5 mRNA expression and PPAR , CCND2 and $A D A M 12$ mRNA expressions at baseline and after the intervention, as well as with the changes of ADAM12, $A D I P O Q$ and NOTCH2 gene expressions. It is possible that the effect of MFAP5 on angiogenesis or the role of MFAP5 gene in AT remodeling is mediated via factors related to adipogenesis and differentiation. It could also be possible that MFAP5 is a 'structure' gene and an 'innocent bystander' that will respond along with other ECM proteins to state of obesity and adipokines secreted by AT. Further studies are necessary to determine which mechanisms might be involved in the regulation of MFAP5 expression in adipocytes.

In conclusion, MFAP5 is highly expressed in AT and is correlated with markers of insulin resistance. It is possible that MFAP5 is involved in ECM remodeling, which enables AT enlargement in obesity. This process seems to be reversible, at least partly, along with WR.

\section{Conflict of interest}

The authors declare no conflict of interest.

\section{Acknowledgements}

We thank Mrs Päivi Turunen for the technical assistance. This work has been financially supported by grants from The Finnish Academy (No. 117844; No. 118590 (MU)), EVO-fund of the Kuopio University Hospital 5254 from Ministry of Health and Social Affairs, TEKES funding (No. 40058/07), Sigrid Juselius Foundation, the Diabetes Research Foundation, Nordic Centre of Excellence on 'Systems biology in controlled dietary interventions and cohort studies (SYSDIET; No. 070014).

\section{References}

1 Clément K, Langin D. Regulation of inflammation-related genes in human adipose tissue. J Intern Med 2007; 262: 422-430.

2 Nair S, Lee YH, Rousseau E, Tataranni PA, Baier LJ, Bogardus C. Increased expression of inflammation-related genes in cultured preadipocytes/stromal vascular cells from obese compared with non-obese Pima Indians. Diabetologia 2005; 48: 1784-1788.

3 Clément K, Viguerie N, Poitou C, Carette C, Pelloux V, Curat CA et al. Weight loss regulates inflammation-related genes in white adipose tissue of obese subjects. FASEB J 2004; 18: 1657-1669.

4 Kolehmainen M, Salopuro T, Schwab U, Kekäläinen J, Kallio P, Laaksonen $\mathrm{D}$ et al. Weight reduction modulates expression of genes involved in extracellular matrix and cell death: the GENOBIN study. Int J Obes 2008; 32: 292-303.

5 Gibson MA, Finnis ML, Kumaratilake JS, Cleary EG. Microfibrilassociated glycoprotein-2 (MAGP-2) is specifically associated with fibrillin-containing microfibrils but exhibits more restricted patterns of tissue localization and developmental expression than its structural relative MAGP-1. J Histochem Cytochem 1998; 46: 871-885.
6 Expert panel on detection, evaluation, treatment of high blood cholesterol in adults. Executive summary of the third report of the National cholesterol education program (NCEP) expert panel on detection, evaluation, and treatment of high blood cholesterol in adults (Adult Treatment Panel III). JAMA 2001; 285: 2486-2497.

7 Sarkkinen E, Schwab U, Niskanen L, Hannuksela M, Savolainen $\mathrm{M}$, Kervinen $\mathrm{K}$ et al. The effects of monounsaturated-fat enriched diet and polyunsaturated-fat enriched diet on lipid and glucose metabolism in subjects with impaired glucose tolerance. Eur J Clin Nutr 1996; 50: 592-598.

8 Boston RC, Stefanovski D, Moate PJ, Summer AE, Watanabe RM, Bergman RN. MINMOD Millennium: a computer program to calculate glucose effectiveness and insulin sensitivity from the frequently sampled intravenous glucose tolerance test. Diabetes Technol Ther 2003; 5: 1003-1015.

9 Miyamoto A, Lau R, Hein PW, Shopley JM, Weinmaster G. Microfibrillar proteins MAGP-1 and MAGP-2 induce Notch1 extracellular domain dissociation and receptor activation. J Biol Chem 2006; 281: 10089-10097.

10 Lemaire R, Bayle J, Mecham RP, Lafyatis R. Microfibril-associated MAGP-2 stimulates elastic fiber assembly. J Biol Chem 2007; 282: 800-808.

11 Spivey KA, Banyard J. A prognostic gene signature in advanced ovarian cancer reveals a microfibril-associated protein (MAGP2) as a promoter of tumor cell survival and angiogenesis. Cell Adh Migr 2010; 4: 169-171.

12 Albig AR, Becenti DJ, Roy TG, Schiemann WP. Microfibrilassociate glycoprotein-2 (MAGP-2) promotes angiogenic cell sprouting by blocking notch signaling in endothelial cells. Microvasc Res 2008; 76: 7-14.

13 Lemaire R, Farina G, Kissin E, Shipley JM, Bona C, Korn J et al. Mutant fibrillin 1 from tight skin mice increases extracellular matrix incorporation of microfibril-associated glycoprotein 2 and type I collagen. Arthritis Rheum 2004; 50: 915-926.

14 Taleb S, Cancello R, Clément K, Lacasa D. Cathepsin s promotes human preadipocyte differentiation: possible involvement of fibronectin degradation. Endocrinology 2006; 147: 4950-4959.

15 Divoux A, Clément K. Architecture and the extracellular matrix: the still unappreciated components of the adipose tissue. Obes Rev 2011; 12: e494-e503.

16 Christiaens V, Lijnen HR. Angiogenesis and development of adipose tissue. Mol Cell Endocrinol 2010; 318: 2-9.

17 Poussin C, Hall D, Minehira K, Galzin A-M, Tarussio D, Thorens B. Different transcriptional control of metabolism and extracellular matrix in visceral and subcutaneous fat of obese and rimonabant treated mice. Plos One 2008; 3: e3385.

18 Henegar C, Tordjman J, Achard V, Lacasa D, Cremer I, GuerreMillo $\mathrm{M}$ et al. Adipose tissue transcriptomic signature highlights the pathological relevance of extracellular matrix in human obesity. Genome Biol 2008; 9: R14.

19 Khan T, Muise E, Iyengar P, Wang Z, Chandalia M, Abate N et al. Metabolic dysregulation and adipose tissue fibrosis: role of collagen VI. Mol Cell Biol 2009; 29: 1575-1591.

20 Pasarica M, Gowronska-Kozak B, Burk D, Remedios I, Hymel D, Gimble $\mathrm{J}$ et al. Adipose tissue collagen VI in obesity. J Clin Endocrinol Metab 2009; 94: 5155-5162.

21 Spencer M, Yao-Borengasser A, Unal R, Rasouli N, Gurley C, Zhu B et al. Adipose tissue macrophages in insulin-resistant subjects are associated with collagen VI and fibrosis and demonstrate alternative activation. Am J Physiol Endocrinol Metab 2010; 299: e1016-e1027.

22 Szauter K, Ordas A, Laxer R, Pope E, Wherrett D, Alman B et al. A novel fibrotic disorder associated with increased dermal fibroblast proliferation and downregulation of genes of the microfibrillar network. Br J Dermatol 2010; 163: 1102-1115.

23 Lemaire R, Korn J, Shipley J, Lafyatis R. Increased expression of type I collagen induced by microfibrillar-associated glycoprotein 2. Arthritis Rheum 2005; 52: 1812-1823. 
24 Nedvídková J, Smitka K, Kopský V, Hainer V. Adiponectin, an adipocyte-derived protein. Physiol Res 2005; 54: 133-140.

25 Phng L-K, Gerhardt H. Angiogenesis: a team effort coordinated by notch. Dev Cell 2009; 16: 196-208.

26 Kawaguchi N, Sundberg C, Kveiborg M, Moghadaszadeh B, Asmar M, Dietrich $\mathrm{N}$ et al. ADAM12 induces actin cytoskeleton and extracellular matrix reorganization during early adipocyte differentiation by regulating beta1 integrin function. J Cell Sci 2003; 116: 3893-3904.

27 Hishida $T$, Naito $K$, Osada $S$, Nishizuka $M$, Imagawa $M$. Crucial roles of D-type cyclins in the early stage of adipo-

cyte differentiation. Biochem Biophys Res Commun 2008; 370: 289-294.

(1)

SOMERाIEHIS RESE

Commons Attribution-NonCommercial-No

Derivative Works 3.0 Unported License. To view a copy

of this license, visit http://creativecommons.org/

licenses/by-nc-nd/3.0/ 